\title{
A General Multichannel Image Restoration Method Using Compound Models
}

\author{
Rafael Molina, Javier Mateos, Aggelos K. Katsaggelos, and Miguel Vega
}

(a) Departamento de Ciencias de la Computación e I.A. Universidad de Granada, 18071 Granada, Spain.

(b) Department of Electrical and Computer Engineering, Northwestern University, Evanston, Illinois 60208-3118.

(c) Departamento de Lenguajes y Sistemas Informáticos, Universidad de Granada, 18071 Granada, Spain.

\begin{abstract}
In this paper we present a multichannel image restoration method using Compound Gauss Markov Random Field (CGMRF) models. Information regarding the objects present in the scene is shared via the line process in the CGMRF. Two new iterative algorithms to estimate the underlying multichannel image are presented, which can be considered as extensions of the classical simulated annealing and ICM methods. Experimental results demonstrate the effectiveness of the proposed approach.
\end{abstract}

\section{Introduction}

In many applications the images to be processed have a multichannel nature; that is, there are several image planes available, called channels, with redundant, as well as complementary information. The different channels may correspond, for instance, to different frequencies, different sensors, or different time frames. The goal of multichannel image restoration is to obtain an estimate of the source multichannel image from its blurred and noisy observation, exploiting the known complementarity of the different channels.

Previous works have approached the multichannel restoration problem by enforcing similarity between intensity values of corresponding pixels in different channels (see [1, 2, 4] and [9] and the references therein). These techniques give results which range from a clear improvement of multichannel over single-channel processing, if the different channel images are highly correlated, to a worsening if they are uncorrelated [2].

In this paper we extend the results of $[5,7]$ to the case when cross-channel degradation is present, providing a general solution to the multichannel image restoration problem by capitalizing on the similarity of edges among he various channels. This similarity is introduced by employing

\footnotetext{
${ }^{1}$ This work has been supported by the "Comisión Nacional de Ciencia y Tecnología” under contract TIC2000-1275.
}

Compound Gauss Markov Random Field (CGMRF) models as priors for the multichannel restoration problem and by favoring the presence of an edge in a channel if the same edge is present in the other channels. This results in detecting regions present in all image channels and successfully preventing color bleeding of image edges while preserving the local characteristics of each channel.

The paper is organized as follows. In section 2 notation, the proposed model for the image and line processes, and the noise model are introduced. In section 3 we present a modified version of the stochastic algorithm and its corresponding deterministic one to restore multichannel images when blurring is present. In section 4, the performance of the proposed algorithms is presented and finally, section 5 concludes the paper.

\section{Notation and Model}

We will distinguish between $f$, the 'true' or source image which would be observed under ideal conditions (i.e., no noise and no distortions produced by blurring and instrumental effects) and $g$, the observed image. Let us assume that there are $L$ channels, each one of size $M \times$ $N$, represented by $f^{t}=\left(f^{1^{t}} f^{2^{t}} \ldots f^{L^{t}}\right)$ and $g^{t}=$

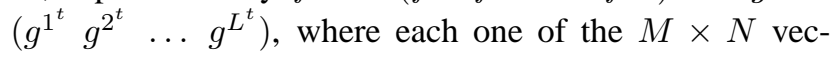
tors $g^{c}, f^{c}, c=1, \ldots, L$, results from the lexicographic ordering of the two-dimensional signal in each channel. We will denote by $f_{i}^{c}$ the intensity of the original channel $c$ at the pixel location $i$ on the lattice. The convention applies equally to the observed image $g$. Our aim is to reconstruct $f$ from $g$. Bayesian methods start with a prior distribution, a probability distribution over images $f$ where we incorporate information on the expected structure within an image. When using a CGMRF as prior distribution, we also introduce a line process, $l$, that, intuitively, acts as an activator or inhibitor of the relation between two neighboring pixels depending on whether or not the pixels are separated by an edge. In the Bayesian framework it is also necessary to specify $\mathrm{p}(g \mid f, l)$ which models how the observed image has been obtained from the 'true' one. The 
Bayesian paradigm dictates that inference about the true $f$ and $l$ should be based on $\mathrm{p}(f, l \mid g)$ given by

$$
\mathrm{p}(f, l \mid g)=\frac{\mathrm{p}(g \mid f, l) \mathrm{p}(f, l)}{\mathrm{p}(g)} \propto \mathrm{p}(g \mid f, l) \mathrm{p}(f, l) .
$$

Maximization of (1) with respect to $f$ and $l$ yields

$$
\hat{f}, \hat{l}=\arg \max _{f, l} \mathrm{p}(f, l \mid g),
$$

the maximum a posteriori (MAP) estimate.

The degradation model relating $g$ and $f$ is given by

$$
g=H f+n,
$$

where $n^{t}=\left(n^{1^{t}} n^{2^{t}} \ldots n^{L^{t}}\right)$ is a Gaussian noise vector of independent components with $n_{i}^{c} \sim N\left(0, \sigma_{\mathrm{n}}^{c^{2}}\right)$. Note that each $n^{c}$ is an $M \times N$ column vector. $H$ is the $[L \times(M \times$ $N)] \times[L \times(M \times N)]$ multichannel blurring matrix of the form

$$
H=\left(\begin{array}{cccc}
\lambda^{11} H^{11} & \lambda^{12} H^{12} & \cdots & \lambda^{1 L} H^{1 L} \\
\lambda^{21} H^{21} & \lambda^{22} H^{22} & \cdots & \lambda^{2 L} H^{2 L} \\
\vdots & \vdots & \ddots & \vdots \\
\lambda^{L 1} H^{L 1} & \lambda^{L 2} H^{L 2} & \cdots & \lambda^{L L} H^{L L}
\end{array}\right)
$$

whose components, $H^{c c^{\prime}}$, are $(M \times N) \times(M \times N)$ matrices defining systematic blurs (assumed to be known) and $\lambda^{c c^{\prime}}$ denotes the contribution of each channel $c^{\prime}$ to the blurring of a given channel $c$. Note, that $\forall c, c^{\prime}, H^{c c^{\prime}}$ is a "classical" energy preserving linear space-invariant blurring function used in single channel restoration problems and that for $H$ to be an energy preserving multichannel blurring matrix, $\lambda^{c c^{\prime}} \geq 0, \forall c, c^{\prime}$, and $\sum_{c^{\prime}=1}^{L} \lambda^{c c^{\prime}}=1, \forall c$. It is important to note that the case studied in $[5,7]$ corresponds to $\lambda^{c c}=1$, $\forall c$ and $\lambda^{c c^{\prime}}=0$, if $c \neq c^{\prime}$.

With the above formulation, each component of $g, g_{i}^{c}$, is obtained as

$$
g_{i}^{c}=(H f)_{i}^{c}+n_{i}^{c}=\sum_{c^{\prime}=1}^{L} \lambda^{c c^{\prime}} \sum_{j} h_{(i-j)}^{c c^{\prime}} f_{j}^{c^{\prime}}+n_{i}^{c},
$$

where $h_{i}^{c c^{\prime}}$ are the coefficients defining the blurring function represented in $H^{c c^{\prime}}$.

We therefore have that the probability of the observed image $g$ if $f$ were the 'true' image and $l$ the 'true' line process is

$$
\mathrm{p}(g \mid f, l) \propto \exp \left\{-\frac{1}{2}(g-H f)^{t} D_{n}(g-H f)\right\},
$$

(note that $\mathrm{p}(g \mid f, l)=\mathrm{p}(g \mid f))$ where $D_{n}$ is an $[L \times(M \times$ $N)] \times[L \times(M \times N)]$ block-diagonal matrix whose entries $D_{n}^{c}, c \in\{1, \ldots, L\}$, are $(M \times N) \times(M \times N)$ diagonal matrices of the form $\beta^{c} I$ with $\beta^{c}=1 / \sigma_{\mathrm{n}}^{c^{2}}$.
The CGMRF prior model we use in this paper is

$$
\begin{gathered}
-\log \mathrm{p}(f, l)=\mathrm{const}+\sum_{c=1}^{L}\left[\frac { 1 } { 2 } \alpha ^ { c } \sum _ { i } \left[(1-4 \phi) f_{i}^{c^{2}}\right.\right. \\
+\phi\left(f_{i}^{c}-f_{i:+1}^{c}\right)^{2}\left(1-l_{[i, i:+1]}^{c}\right)+\tau^{c} l_{[i, i:+1]}^{c} \\
+\phi\left(f_{i}^{c}-f_{i:+2}^{c}\right)^{2}\left(1-l_{[i, i:+2]}^{c}\right)+\tau^{c} l_{[i, i:+2]}^{c} \\
\left.-\sum_{\substack{c^{\prime}=1 \\
c^{\prime} \neq c}}^{L} \epsilon^{c c^{\prime}}\left(l_{[i, i:+1]}^{c} l_{[i, i:+1]}^{c^{\prime}}+l_{[i, i:+2]}^{c} l_{[i, i:+2]}^{c^{\prime}}\right]\right],(5)
\end{gathered}
$$

where we are assuming a 'toroidal edge correction', $i:+1$, $i:+2, i:+3, i:+4$ denote the four pixels around pixel $i$ (if $i=(u, v)$ they correspond to $(u+1, v),(u, v+1),(u-$ $1, v)$, and $(u, v-1)$, respectively) and $l_{[i, j]}^{c}$ denotes the line process between pixels $i$ and $j$ in channel $c$, which takes the value one if they are separated by an active line and zero otherwise. We then penalize the introduction of an active line element in the position $[i, j]$ with the term $\tau^{c} l_{[i, j]}^{c}$. By introducing the term $\left.\epsilon^{c c^{\prime}}\left(l_{[i, i:+1]}^{c}\right]_{[i, i:+1]}^{c^{\prime}}+l_{[i, i:+2]}^{c} l_{[i, i:+2]}^{c^{\prime}}\right)$ with $c, c^{\prime}=1, \ldots, L, c \neq c^{\prime}$ and $\epsilon^{c c^{\prime}} \geq 0$, we increase the probability of a new active line element in the position $[i, j]$ in a given channel if the other channels have a line in the same position. The introduction of these cross terms will help to recognize the same objects in all the channels even if they do not have similar intensities. The parameter $\alpha^{c}$ measures the smoothness of the 'true' channel $c$ and $\phi$ has to be less than 0.25 .

\section{MAP estimation using Stochastic and Deter- ministic Relaxation}

In order to find the MAP estimate for this problem where the prior model explicitly favors the presence of discontinuities, we will use a modified version of the simulated annealing (SA) algorithm (see [3] and also [6]) which, for our multichannel MAP estimation problem defined in (2), uses the probability distribution, derived from (4) and (5),

$$
\begin{aligned}
\mathrm{p}_{T}(f, l \mid g) \propto \exp \left\{-\frac{1}{T}\left[\frac{1}{2}(g-H f)^{t} D_{n}(g-H f)\right.\right. \\
+\sum_{c=1}^{L} \frac{1}{2} \alpha^{c} \sum_{i}\left[(1-4 \phi) f_{i}^{c^{2}}\right. \\
+\phi\left(f_{i}^{c}-f_{i:+1}^{c}\right)^{2}\left(1-l_{[i, i:+1]}^{c}\right)+\tau^{c} l_{[i, i:+1]}^{c} \\
+\phi\left(f_{i}^{c}-f_{i:+2}^{c}\right)^{2}\left(1-l_{[i, i:+2]}^{c}\right)+\tau^{c} l_{[i, i:+2]}^{c} \\
\left.\left.-\sum_{\substack{c^{\prime}=1 \\
c^{\prime} \neq c}}^{L} \epsilon^{c c^{\prime}}\left(l_{[i, i:+1]}^{c} l_{[i, i:+1]}^{c^{\prime}}+l_{[i, i:+2]}^{c} l_{[i, i:+2]}^{c^{\prime}}\right]\right]\right\},
\end{aligned}
$$




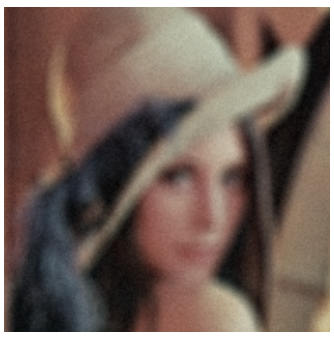

a
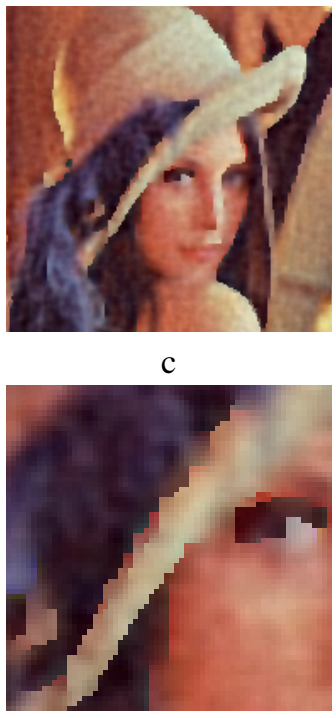

e

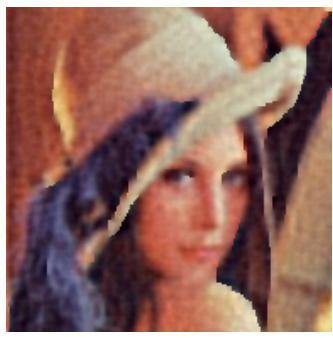

b

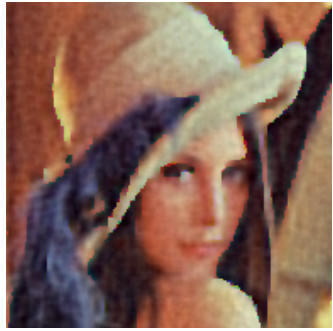

d

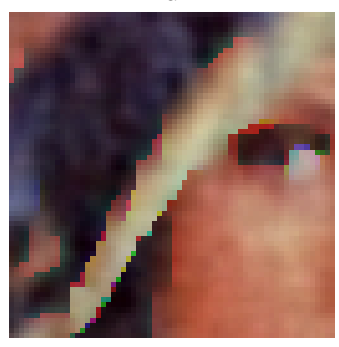

$\mathrm{f}$
Figure 1. (a) Degraded image; Restored images by (b) the MMICM method. (c) the MMSA method. (d) the MMSA method with $\epsilon^{c c^{\prime}}=0, \forall c, c^{\prime}$. (e) Enlarged part of (c). (f) Enlarged part of (d).

where $T$ is the temperature. The algorithm simulates the conditional a posteriori density function of $l_{[i, j]}^{c}$, given the rest of $l, f$ and $g$ and the conditional a posteriori density function of $f_{i}^{c}$ given the rest of $f, l$ and $g$.

To simulate $\mathrm{p}_{T}\left(l_{[i, j]}^{c} \mid l_{[m, n]}^{c}:[m, n] \neq[i, j], l^{c^{\prime}}: c^{\prime} \neq\right.$ $c, f, g)$, we have

$$
\begin{array}{r}
\mathrm{p}_{T}\left(l_{[i, j]}^{c}=0 \mid l_{[m, n]}^{c}:[m, n] \neq[i, j], l^{c^{\prime}}: c^{\prime} \neq c, f, g\right) \\
\propto \exp \left[-\frac{1}{T} \frac{\alpha_{c} \phi}{2}\left(f_{i}^{c}-f_{j}^{c}\right)^{2}\right], \\
\mathrm{p}_{T}\left(l_{[i, j]}^{c}=1 \mid l_{[m, n]}^{c}:[m, n] \neq[i, j], l^{c^{\prime}}: c^{\prime} \neq c, f, g\right) \\
\propto \exp \left[-\frac{1}{T} \frac{\alpha_{c}}{2}\left(\tau^{c}-\sum_{c^{\prime} \neq c} \epsilon^{c c^{\prime}} l_{[i, j]}^{c^{\prime}}\right)\right] .
\end{array}
$$

To simulate the conditional a posteriori density function of $f_{i}^{c}$ given the rest of $f, l$ and $g$, we have

$$
\mathrm{p}_{T}\left(f_{i}^{c} \mid f_{j}^{c}: \forall j \neq i, f^{c^{\prime}}: \forall c^{\prime} \neq c, l, g\right) \sim \mathcal{N}\left(\mu_{i}^{c}, T \sigma_{i}^{c^{2}}\right),
$$

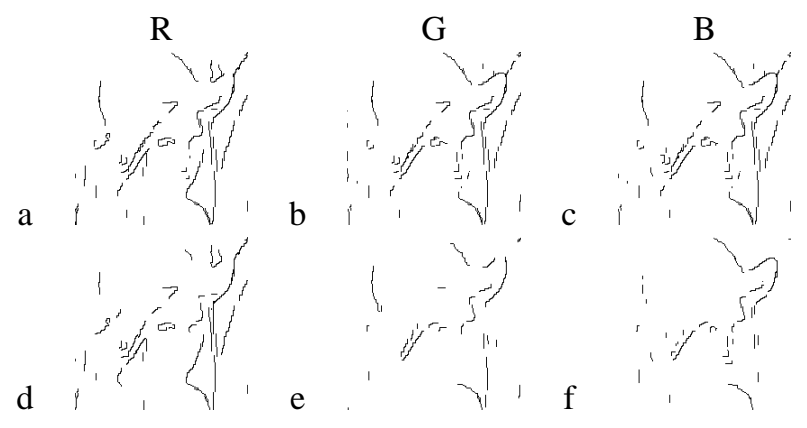

Figure 2. Line process for (a-c) Fig. 1c, (d-f) Fig. 1d.

where

$$
\begin{aligned}
\mu_{i}^{c}= & f_{i}^{c}-\rho\left\{\alpha^{c} \phi \sum_{j \operatorname{nhbr} i}\left(f_{i}^{c}-f_{j}^{c}\right)\left(1-l_{[i, j]}^{c}\right)\right. \\
& \left.+(1-4 \phi) f_{i}^{c}-\left[H^{t} D_{n}(g-H f)\right]_{i}^{c}\right\}
\end{aligned}
$$

and

$$
\sigma_{i}^{c^{2}}=\left(1-\left(1-\tau_{i}^{c} / \rho\right)^{2}\right) / \tau_{i}^{c},
$$

where $\rho=1 / \max \left(\alpha^{c}+\sum_{c^{\prime}} \beta^{c^{\prime}} \lambda^{c^{\prime} c}\right), \tau_{i}^{c}=\alpha^{c} n n_{i}^{c}(l)+$ $\mathrm{s}^{c}, n n_{i}^{c}(l)=\phi \sum_{j \text { nhbr } i}\left(1-l_{[i, j]}^{c}\right)+(1-4 \phi)$, the suffix ' $j$ nhbr $i$ ' denotes the four neighboring pixels at distance one from pixel $i$, and $\mathrm{s}^{c}=\sum_{c^{\prime}=1}^{L} \beta^{c^{\prime}} \lambda^{c^{\prime} c^{2}} \sum_{i} h_{i}^{c^{\prime} c^{2}}$.

Having defined the required probabilities, the resulting proposed Multichannel Modified Simulated Annealing algorithm (MMSA) proceeds as follows:

\section{Algorithm 1 (MMSA procedure)}

1. Set $t=0$ and assign an initial configuration denoted as $f^{(-1)}, l^{(-1)}$ and initial temperature $T^{(0)}=1$.

2. The evolution $l^{(t-1)} \rightarrow l^{(t)}$ of the line process is obtained by sampling the next point of the line process (using rasterscanning scheme) based on the conditional probability mass functions defined in (7) and (8) and keeping the rest of $l^{(t-1)}$ unchanged.

3. Set $t=t+1$. Go back to step 2 until a complete sweep of the field $l$ is finished.

4. The evolution $f^{(t-1)} \rightarrow f^{(t)}$ of the image $f$ can be obtained by sampling the next value of the whole image based on the conditional probability mass function given in (9).

5. Set $T=C_{T} / \log (1+k(t))$, where $C_{T}$ is a constant and $k(t)$ is the sweep number at iteration number $t$.

6. Go to step 2 until $t>t_{f}$, where $t_{f}$ is a specified value.

Instead of using a stochastic approach, we can use a deterministic method to search for a local maximum. An advantage of the deterministic method is that its convergence is much faster than that of the stochastic approach, since instead of simulating the distributions, the mode from the corresponding conditional distribution is chosen, hence the name Iterative Conditional Mode (ICM).

In [8] we prove that both the MMSA and the Modified Multichannel ICM (MMICM) algorithms converge to a local MAP estimate, even in the presence of blurring. 


\section{Experimental Results}

Experiments were carried out with RGB color images in order to evaluate the performance of the proposed methods. Although visual inspection of the restored images is a very important quality measure for the restoration problem, in order to get quantitative image quality comparisons, the peak signal-to-noise ratio $(P S N R)$ of each channel is used. Given two image channels $f^{c}$ and $g^{c}$ of size $M \times N$ and values in the range $[0,255]$, the $P S N R$ of channel $c$ is defined as

$$
P S N R=10 \log _{10}\left[\frac{M \times N \times 255^{2}}{\left\|g^{c}-f^{c}\right\|^{2}}\right] .
$$

The mean of the $P S N R$ values of all channels is used as a figure of merit for the color image.

Results are reported on the $256 \times 256$ "Lena" image which was blurred with a color bleeding multichannel point spread function, $H$, given by

$$
H=\left(\begin{array}{ccc}
0.7 H^{0} & 0.2 H^{0} & 0.1 H^{0} \\
0.1 H^{0} & 0.7 H^{0} & 0.2 H^{0} \\
0.2 H^{0} & 0.1 H^{0} & 0.7 H^{0}
\end{array}\right)
$$

where $H^{0}$ defines an out-of-focus blur with radius 5 . Gaussian noise of variance $\sigma_{\mathrm{n}}^{c^{2}}=16$ was added to each channel, $c$, resulting in the degraded image shown in Fig. 1a (only an $192 \times 192$ central part is shown).

The MMSA and MMICM algorithms were run on this multichannel image with the parameters for the prior model experimentally chosen to be $\alpha^{c}=1 / 150, \tau^{c}=260$ and $\epsilon^{c c^{\prime}}=105$, for all $c$ and $c^{\prime}$. The $192 \times 192$ central part of the restored images by the MMICM and MMSA algorithms are shown in Fig. $1 \mathrm{~b}$ and $1 \mathrm{c}$, respectively. The $P S N R$ values corresponding to the whole images are summarized in table 1. These results show that both the MMICM and the MMSA algorithms produce very good restorations both in terms of visual quality and $P S N R$ values; the mean $P S N R$ value for the MMSA algorithm is slightly better. The effect of the cross terms, $\epsilon^{c c^{\prime}}\left(l_{[i, i:+1]}^{c}\right]_{[i, i:+1]}^{c^{\prime}}+$ $\left.l_{[i, i:+2]}^{c} l_{[i, i:+2]}^{c^{\prime}}\right)$, introduced in Eq. (5) is clear by looking at Fig. 1d and its enlargement in Fig. 1f, where these cross terms are not considered $\left(\epsilon^{c c^{\prime}}=0, \forall c, c^{\prime}\right)$. Note that the edge of the hat and the white of the eye show color bleeding. This effect is clearly corrected by introducing the cross terms as shown in Fig. 1c and its enlargement (Fig. 1e). The line processes, shown in Fig. 2, also reflect this improvement. Note, however, that the difference in $P S N R$ (Table 1) is very small due to the small number of pixels affected by this change in the line process. The $P S N R$ is consistently higher when the cross terms are considered.
Table 1. $P S N R$ for the different restorations.

\begin{tabular}{|l|c|c|c|c|}
\hline$P S N R(\mathrm{~dB})$ & red & green & blue & mean \\
\hline Observed & 18.593 & 20.286 & 21.146 & 20.008 \\
\hline MMSA & 24.194 & 24.133 & 24.835 & 24.387 \\
\hline MMICM & 24.154 & 24.075 & 24.843 & 24.357 \\
\hline $\begin{array}{l}\text { MMSA } \\
\left(\epsilon^{c c^{\prime}}=0, \forall c, c^{\prime}\right)\end{array}$ & 24.018 & 23.945 & 24.750 & 24.237 \\
\hline
\end{tabular}

\section{Conclusions}

In this paper we have used CGMRF prior models in multichannel image restoration problems. The exchange of information among channels is carried out through the line process which preserves similar regions in the channels. In order to find the MAP estimates for these problems we have proposed two new methods that can be considered as extensions of the classical ICM and SA procedures. The experimental results show improvement on the restoration of multichannel images based both on visual and PNSR criteria.

\section{References}

[1] N. P. Galatsanos and R. T. Chin. Digital restoration of multichannel images. IEEE Trans. on Acoustic, Speech and Signal Proc., 37(3):415-421, 1989.

[2] N. P. Galatsanos, A. K. Katsaggelos, R. T. Chin, and A. D. Hillery. Least squares restoration of multichannel images. IEEE Trans. on Signal Proc., 39(10):2222-2236, 1991.

[3] S. Geman and D. Geman. Stochastic relaxation, Gibbs distributions, and the Bayesian restoration of images. IEEE Trans. on Pattern Analysis and Machine Intell., 9(6):721-742, 1984.

[4] M. G. Kang. Generalized multichannel image deconvolution approach and its applications. Optical Eng., 37:2953-2964, 1998.

[5] J. Mateos, R. Molina, and A. K. Katsaggelos. Color image restoration using compound Gauss-Markov random fields. In X European Signal Proc. Conf. (EUSIPCO'2000), volume III, pages 1341-1344, 2000.

[6] R. Molina, A. K. Katsaggelos, J. Mateos, A. Hermoso, and C. A. Segall. Restoration of severely blurred high range images using stochastic and deterministic relaxation algorithms in compound Gauss Markov random fields. Pattern Recogn., 33(3):557-571, 2000.

[7] R. Molina, J. Mateos, and A. K. Katsaggelos. Multichannel image restoration using compound Gauss-Markov random fields. In Int. Conf. on Acoustics, Speech, and Signal Proc., (ICASSP 2000), volume 1, pages 141-144, 2000.

[8] R. Molina, J. Mateos, A. K. Katsaggelos, and M. Vega. Bayesian multichannel image restoration using compound Gauss-Markov random fields. Submitted to IEEE Trans. on Image Proc., 2001.

[9] F. Sroubek and J. Flusser. An overview of multichannel image restoration techniques. In J. Safrànkovà, editor, Week of Doctoral Students 1999, pages 580-585. Matfyzpress, Praha, 1999. 